Yang, R., Jin, L., Paternain, D., Yager, R. R., Mesiar, R., \& Bustince, H. (2020). Some preference involved aggregation models for basic uncertain information using uncertainty transformation. Journal of Intelligent \& Fuzzy Systems, 39(1), 325-332. https://doi.org/10.3233/JIFS-191106

Journal of Intelligent \& Fuzzy Systems xx (20xx) x-xx

DOI:10.3233/JIFS-191106

IOS Press

\title{
Some preference involved aggregation models for basic uncertain information using uncertainty transformation
}

\author{
RouJian Yang ${ }^{\mathrm{a}}$, LeSheng Jin ${ }^{\mathrm{b}, *}$, Daniel Paternain ${ }^{\mathrm{c}}$, Ronald R. Yager ${ }^{\mathrm{d}}$, Radko Mesiar ${ }^{\mathrm{e}}$ \\ and Humberto Bustince \\ ${ }^{a}$ School of Government Audit, Nanjing Audit University, Nanjing, China \\ ${ }^{\mathrm{b}}$ Business School, Nanjing Normal University, Nanjing, China \\ ${ }^{\mathrm{c}}$ Department of Statistics, Computer Science and Mathematics, Public University of Navarre, Campus Arrosadia \\ sn, Pamplona, Spain \\ ${ }^{\mathrm{d}}$ Machine Intelligence Institute, Iona College, New Rochelle, NY, USA \\ ${ }^{\mathrm{e}}$ Faculty of Civil Engineering, Slovak University of Technology, Slovakia, Palacky Univ Olomouc, Fac Sci, Dept \\ Algebra \& Geometry, Olomouc, Czech Republic \\ ${ }^{\mathrm{f}}$ Department of Statistics, Computer Science and Mathematics, Public University of Navarre, Campus Arrosadia \\ sn, Pamplona, Spain
}

\begin{abstract}
In decision making, very often the data collected are with different extents of uncertainty. The recently introduced concept, Basic Uncertain Information (BUI), serves as one ideal information representation to well model involved uncertainties with different extents. This study discusses some methods of BUI aggregation by proposing some uncertainty transformations for them. Based on some previously obtained results, we at first define IOWA operator with poset valued input vector and inducing vector. The work then defines the concept of uncertain system, on which we can further introduce the multi-layer uncertainty transformation for BUI. Subsequently, we formally introduce MUT_IOWA aggregation procedure, which has good potential to more and wider application areas. A numerical example is also offered along with some simple usage of it in decision making.
\end{abstract}

Keywords: Aggregation function, BUI aggregation, decision making, evaluation, OWA operators, uncertain decision making

\section{Introduction}

Evaluations methods and theories are cornerstones of decision making models and practices. The applications and developments of aggregation functions (also known as aggregation operators) [1, 3, 11, 20] and information fusion techniques [4, 9, 13, 23-26] play an underpinning role in a myriad of evaluation

${ }^{*}$ Corresponding author. LeSheng Jin, Business School, Nanjing Normal University, Nanjing, China. E-mail: j1s1980@163.com. problems $[12,14,15,21]$. For several decades, scholars deeply and widely have been studying aggregation functions from different aspects $[3,6-8,10,16,17$, $19,25]$. In general, given a collection of finite pieces of information under evaluation and aggregation, aggregation functions always take those information as inputs and then return an aggregated result; and the result often serves as a comprehensive evaluation to a related decision making problem.

There are numerous different types and classifications of aggregation functions such as averaging 
functions, conjunctive functions, disjunctive functions and mixture functions [3, 10, 11]. A type of powerful and important aggregation function is the Ordered Weighted Averaging (OWA) operators [22], which can flexibly and effectively model a continuum bipolar preference from optimism, via neutral attitude, to pessimism of decision makers, taken or exerted over inputs information. One important extension of OWA operators is the Induced Ordered Weighted Averaging (IOWA) operators [24], endowed with further flexibility to well embody and reflect a wider type of bipolar preference of decision makers than the mere optimism/pessimism preference.

Viewing further into some atomic structures in this interesting and important research area, scholars and decision makers are faced with great diversity of data information. One hot area of research of analyzing and modeling for those different types of data, is around the data information that has an uncertainty nature involved. There has been a large variety of different types of uncertain information such as the well known fuzzy information [2], interval information, probability information, possibility information and recently introduced Basic Uncertain Information (BUI) $[5,18]$. Put simply, a BUI granule is with the form $\langle x ; c\rangle(x, c \in[0,1])$, where $x$ is the input value as normally dealt with under aggregation, while $c$ is the certainty degree of $x$, which will be reviewed in detail later. Actually, many uncertainties can be generalized into BUI, since there needs only one value in unit interval to efficiently and effectively model the certainty degree a decision maker has.

It is evident that the existence of uncertainties will cause those involved decision makers to have different cognitions and more complex evaluations over the inputs information. Accordingly, the traditional preference aggregation techniques should be adapted or adjusted to address the new problems posed. And as an essential task, scholars and practitioners should consider how to better model the uncertainties existing in different forms, and devise suitable aggregation procedures to merge those uncertain information (e.g., with BUI expression) and take corresponding and reasonable decisions. For these purposes, this study will analyze and discuss some reasonable preference aggregation techniques that are specifically suitable for BUI inputs and thus help to provide evaluation and decision taking guidance or automatic decision rules in corresponding uncertain decision making environments.
The remainder of this article is organized as follows. Section 2 reviews some basic knowledge about aggregation function, OWA operators and IOWA operators, fixes some terminologies used in this work, and then formally introduces IOWA operator with poset valued input vector and inducing vector for later discussions. In Section 3, based on well-defined uncertain system, we majorly discuss the multi-layer uncertainty transformation for BUI with corresponding OWA aggregation, and then propose MUT_IOWA aggregation procedure. Section 4 provides a numerical example of MUT_IOWA with simple application in evaluation. Section 5 concludes and remarks this study.

\section{Generalized IOWA aggregation with both poset input and inducing values}

Some evaluation and aggregation operators and techniques that are based on strict formulations, as well as on parameterization and adjustability, are very crucial and helpful in automatic decision making and some corresponding areas of computational intelligences. This section is designed to review, rephrase or reformulate some preference involved aggregation techniques using systematical and formal language.

Without loss of generality, in this study any collection of $n$ pieces of inputs information to be aggregated is represented by a real function $x^{<n>}:\{1, \ldots, n\} \rightarrow$ $[0,1]$, called input function. The space of such input functions (input vectors) $x^{<n>}$ is conventionally denoted by $[0,1]^{n}$. Throughout the rest of this work, we make no difference between an input function and its vector expression.

Definition 1. [3] (Aggregation function) An aggregation function with input vector $x^{<n>}$, $F^{<n>}:[0,1]^{n} \rightarrow[0,1]$ satisfies the following two conditions

(i) (boundary conditions) $F^{<n>}((0, \ldots, 0))=0$ and $F^{<n>}((1, \ldots, 1))=1$;

(ii) (monotonicity) for any two input functions $x^{<n>}, y^{<n>} \in[0,1]^{n}$, if $x^{<n>} \leq y^{<n>}$ then $F^{<n>}\left(x^{<n>}\right) \leq F^{<n>}\left(y^{<n>}\right)$.

Aggregation functions provide a useful and strict frame to handle a wide variety of evaluation and information fusion problems. In decision making, sometimes decision makers have more or less subjectivities and preferences involved. In order to well model the aggregation with optimism/pessimism 5 , , 98 99 100 101 102 103 104 105 106 107 8

.
1 3 政 . . . .

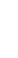
. 24 . 
preferences involved, Yager introduced a special type of aggregation functions, the OWA operators, which can ideally return a larger (or smaller) value than many other types of aggregation functions according to the extents of those involved optimism/pessimism preferences of decision makers.

Definition 2. [22] (OWA operator) Let $w^{<n>}$ : $[0,1]^{n} \rightarrow[0,1]$ be a weight vector (of dimension $n)$ with $\sum_{i=1}^{n} w^{<n>}(i)=1$. An OWA operator of dimension $n$ with weight vector $w^{<n>}$ is defined to be a mapping $O W A_{w^{<n>}}^{<n>}:[0,1]^{n} \rightarrow[0,1]$ such that

$O W A_{w^{<n>}}^{<n>}\left(x^{<n>}\right)=\sum_{i=1}^{n} w^{<n>}(i) x^{<n>}\left(\sigma^{<n>}(i)\right)$

where $\sigma^{<n>}:\{1, \ldots, n\} \rightarrow\{1, \ldots, n\}$ is any appropriate permutation satisfying $x^{<n>}\left(\sigma^{<n>}(i)\right) \geq$ $x^{<n>}\left(\sigma^{<n>}(j)\right)$ whenever $1 \leq i<j \leq n$.

Definition 3. [22] (Orness/andness) The orness of a weight vector (of dimension $n$ ) $w^{<n>}$ is defined by

$$
\operatorname{orness}\left(w^{<n>}\right)=\sum_{i=1}^{n} \frac{n-i}{n-1} w^{<n>}(i)
$$

Dually, the andness of a weight vector (of dimension $n) w^{<n>}$ is defined by

$$
\begin{aligned}
\operatorname{andness}\left(w^{<n>}\right) & =\sum_{i=1}^{n} \frac{i-1}{n-1} w^{<n>}(i) \\
& =1-\operatorname{orness}\left(w^{<n>}\right)
\end{aligned}
$$

In many decision making practices, orness of a weight vector generally reflects the extent to which decision makers have an optimistic preference over the inputs. Ordinarily, the OWA operator with a weight vector having larger orness will return a larger aggregation result, and vice versa. It is possible that the OWA operator with a weight vector having a larger orness can have a smaller value than those having a smaller orness. However, statistically, it is more possible for the OWA aggregation related to larger orness to be greater than the one related to smaller orness. Considering expected values, orness is just the normed expected value of OWA aggregation [3].

Scholars discussed different methods of generating weight vector for OWA operators. Yager proposed an ingenious one that can simply and effectively generate weight vectors by a bounded function $Q$ : $[0,1] \rightarrow[0,1]$ called Regular Increasing Monotone (RIM) quantifier.
Definition 4. [23] A RIM quantifier $Q$ is a monotonic non-decreasing function defined on unit interval $Q:[0,1] \rightarrow[0,1]$ with $Q(0)=0$ and $Q(1)=1$. A weight vector $w_{Q}^{<n>}$ is called a $Q$-generated weight vector (of dimension $n$ ) if it satisfies

$$
w_{Q}^{<n>}(i)=Q(i / n)-Q((i-1) / n)
$$

Remark. Since $Q$ is monotonic non-decreasing, then it is necessarily Riemann Integrable. The orness [23] of such RIM quantifier $Q$ is defined as

$$
\operatorname{orness}(Q)=\int_{0}^{1} Q(t) d t
$$

The orness of RIM quantifier $Q$ is generally not equal to the orness of the derived weight vector $w_{Q}^{<n>}$, though when $n \rightarrow \infty$ they are approaching each other. Similar to the meaning of orness of a weight vector, the orness of a RIM quantifier $Q$ also effectively reflects an extent of optimism of decision makers.

In general, OWA operators can well model only the optimism/pessimism preference when fusing information. Hence, to be able to conveniently model more cases of bipolar preference, Yager later introduced the Induced Ordered Weighted Averaging (IOWA) operators. We rephrase it using the language of RIM quantifier as follows with some more accurate expressions.

Definition 5. [24] (IOWA operator) Given RIM quantifier $Q$, input vector $x^{<n>} \in[0,1]^{n}$, let $h^{<n>} \in$ $[0,1]^{n}$ be another input vector attached to $x^{<n>}$, called the inducing vector attached to $x^{<n>}$, where each of its value $h^{<n>}(i)$ is called the inducing value of $x^{<n>}(i)(i \in\{1, \ldots, n\})$. Define two set functions $L:\{1, \ldots, n\} \rightarrow 2^{\{1, \ldots, n\}}$ and $U:\{1, \ldots, n\} \rightarrow$ $2^{\{1, \ldots, n\}}$ such that

$$
\begin{aligned}
& L(i)=\left\{r \in\{1, \ldots, n\} \mid h^{<n>}(r)<h^{<n>}(i)\right\}, \\
& U(i)=\left\{r \in\{1, \ldots, n\} \mid h^{<n>}(i)<h^{<n>}(r)\right\} .
\end{aligned}
$$

Generate a weight vector from $Q$ and $h^{<n>}, v_{Q ; h}^{<n>}: \quad 206$ $\{1, \ldots, n\} \rightarrow[0,1]$ by the following rule

$v_{Q ; h}^{<n>}(i)=\frac{Q((n-|L(i)|) / n)-Q(|U(i)| / n)}{n-|L(i)|-|U(i)|}$.

An Induced OWA operator $I O W A_{Q ; h}:[0,1]^{n} \rightarrow \quad 209$ $[0,1]$ (with $Q$ and $h^{<n>}$ ) is defined as

$$
\operatorname{IOWA}_{Q ; h}(x)=\sum_{i=1}^{n} v_{Q ; h}^{<n>}(i) \cdot x^{<n>}(i) .
$$


Remark. It is obvious that $v_{Q ; h}^{<n>}$ is indeed a normalized weight vector.

Definition 6. (a) For a poset $(S, \preceq)$, let $H^{<n>}$ : $\{1, \ldots, n\} \rightarrow S$ be function, then denote by $S^{n}$ the space of all such functions $H^{<n>}$.

(b) For any two elements $x, y \in S, x \prec y$ means $x \preceq y$ and $x \neq y$.

We next formally define the IOWA operator with poset valued input vector and inducing vector.

Definition 7. (IOWA operator with poset valued input vector and inducing vector) Given RIM quantifier $Q$, convex poset $\left(S_{1}, \preceq\right)$ (i.e., for any $X_{1}, X_{2} \in S_{1}$, and any $\lambda \in[0,1]$, then $\left.\lambda \cdot X_{1}+(1-\lambda) \cdot X_{2} \in S_{1}\right)$, and poset $\left(S_{2}, \preceq\right)$, let $X^{<n>} \in S_{1}^{n}$ be the convex poset valued input vector, let $H^{<n>} \in S_{2}^{n}$ be the poset valued inducing vector attached to $X^{<n>}$, called the poset valued inducing vector attached to $X^{<n>}$, where each of its value $H^{<n>}(i)$ is called the inducing poset value of $X^{<n>}(i)$. Define three set-valued functions $L$ : $\{1, \ldots, n\} \rightarrow 2^{\{1, \ldots, n\}}, U:\{1, \ldots, n\} \rightarrow 2^{\{1, \ldots, n\}}$ and $E:\{1, \ldots, n\} \rightarrow 2^{\{1, \ldots, n\}}$ such that

$$
\begin{gathered}
L(i)=\left\{r \in\{1, \ldots, n\} \mid H^{<n>}(r) \prec H^{<n>}(i)\right\}, \\
U(i)=\left\{r \in\{1, \ldots, n\} \mid H^{<n>}(i) \prec H^{<n>}(r)\right\}, \text { and }
\end{gathered}
$$

$E(i)=\left\{r \in\{1, \ldots, n\} \mid H^{<n>}(i) \nprec H^{<n>}(r)\right.$ and

$\left.H^{<n>}(r) \nprec H^{<n>}(i)\right\}$

Generate a weight vector from $Q$ and $H^{<n>}$, $v_{Q ; H}^{<n>}:\{1, \ldots, n\} \rightarrow[0,1]$ by the following rule

$v_{Q ; H}^{<n>}(i)=\frac{Q((n-|L(i)|) / n)-Q(|U(i)| / n)}{|E(i)|}$.

An Induced OWA (IOWA) operator with poset valued inducing vector, $I O W A_{Q ; H}:[0,1]^{n} \rightarrow[0,1]$ (with $Q$ and $H^{<n>}$ ) is defined as

$$
\operatorname{IOWA}_{Q ; H}(X)=\sum_{i=1}^{n} w(i) X^{<n>}(i)
$$

where $w$ satisfies $w(i)=\frac{v_{Q ; H}^{<n>>}(i)}{\sum_{j=1}^{n} v_{Q ; H}^{<n>(j)}}$.

Remark. Note that $v_{O: H}^{<n>}$ in (8) is not necessarily normalized, and from [8] we know $w$ in (9) always can be correctly obtained.

Remark. Observe that if $\left(S_{1}, \preceq\right)=\left(S_{2}, \preceq\right)=$ $([0,1], \leq)$, then Definition 7 in actual degenerates into the OWA operators in Definition 2. In other words, an OWA operator is equivalent to an IOWA operator with both of its input vector and inducing vector being real.

239

240

\section{Certainty preferences induced aggregation for BUI with related certainty-transformation}

Recently, a concept of the Basic Uncertain Information (BUI) to generalize and represent a diverse variety of uncertain information was proposed in $[5,18]$. This section discusses two methods to perform OWA aggregation over BUI inputs.

\subsection{The simple uncertainty transformation for BUI}

A BUI granular information is represented as a pair form $\langle x ; c\rangle(x, c \in[0,1])$, where $x$ is the input value under further aggregation, while $c$ is the certainty degree of $x$, representing the extent to which the involved decision makers believe that $x$ takes exactly its value; and $1-c$ is then called the uncertainty degree of $x$. The decision makers' beliefs or confidences of the input value being $x$ can be measured by a value $c$ in unit interval; that is, the larger certainty degree $c$ is, the more beliefs they have, and vice versa. For example, $\langle x ; c\rangle=<0.6,0.3\rangle$ indicates that the input value for aggregation is given with $x=0.6$, but decision maker does not fully believe $x$ assumes 0.6 , and his/her belief for this proposition is $c=0.3$ (and his uncertainty for this is therefore $c=0.7$ ), showing some suspicious feeling of him/her for the proposal $x=0.6$.

When there is need to aggregate a collection of $n$ BUI inputs $\left.\left(<x_{i} ; c_{i}\right\rangle\right)_{i=1}^{n}$ using OWA operator, clearly we also must to consider the influences on $\left(x_{i}\right)_{i=1}^{n}$ exerted by $\left(c_{i}\right)_{i=1}^{n}$. If we directly use IOWA operator with inducing function $h^{<n>}:\{1, \ldots, n\} \rightarrow$ $[0,1]$ such that $h^{<n>}(i)=c_{i}$, then the preference involved aggregation will no longer consider the proposed magnitudes of inputs $\left(x_{i}\right)_{i=1}^{n}$, but by the attached inducing value $h^{<n>}(i)=c_{i}(i \in\{1, \ldots, n\})$ only. It is related to the process of IOWA aggregation, not the desired OWA aggregation. Therefore, in order to fulfill OWA aggregation over BUI inputs $\left(<x_{i} ; c_{i}>\right)_{i=1}^{n}$, we need to make clear that the aggregation is mainly controlled by inputs $\left(x_{i}\right)_{i=1}^{n}$, and inducing information $\left(c_{i}\right)_{i=1}^{n}$ is only the influential factors.

With given inducing information $\left(c_{i}\right)_{i=1}^{n}$, different decision makers may have different feelings and 
opinions about how they will influence the whole OWA aggregation process. These cognitions may also change depending on different decisional situations and scenarios. In the remainder of this study, based on different mechanisms of uncertainty transformation, we will propose some reasonable methods to model those opinions of decision makers, which are meaningful and helpful in the understanding and studying of uncertain decision making.

The next one is about one simple but effective uncertainty transformation for BUI.

Definition 8. (i) The space of all BUI $\langle x ; c\rangle$ is denoted by $\mathcal{B}$; the space of all closed intervals $[a, b]$ $([a, b] \subseteq[0,1])$ is denoted by $\mathcal{I}$. In addition, when there is no confusion arising, $[a, a]$ equivalently represents real number $a$ in decision making.

(ii) Define $(\mathcal{I} ; \preceq)$ to be a poset (actually a complete lattice) such that for any two $\left[a_{1}, b_{1}\right],\left[a_{2}, b_{2}\right] \in \mathcal{I}$, $\left[a_{1}, b_{1}\right] \preceq\left[a_{2}, b_{2}\right]$ if and only if $a_{1} \leq a_{2}, b_{1} \leq b_{2}$.

Definition 9. For any BUI $<x ; c>$, the simple uncertainty transformation $S P: \mathcal{B} \rightarrow \mathcal{I}$ is a mapping such that

$$
S P(<x ; c>)=[c x, x+(1-c)(1-x)] .
$$

With the obtained intervals by uncertainty transformation, the OWA aggregation for BUI inputs $\left(<x_{i} ; c_{i}>\right)_{i=1}^{n}$ boils down to the IOWA operator with poset valued input vector and inducing vector. Specifically, under this situation, the involved input vector $x^{<n>}$ is simply synchronized with $\left(x_{i}\right)_{i=1}^{n}$, i.e., $x^{<n>}(i)=x_{i}$, while the attached poset valued inducing vector $H^{<n>}$ is defined by $H^{<n>}(i)=S P(<$ $\left.x_{i} ; c_{i}>\right)$.

It is noteworthy that above induced aggregation has relation to both $\left(x_{i}\right)_{i=1}^{n}$ and $\left(c_{i}\right)_{i=1}^{n}$, but it does not directly depend on them. The detailed procedures of OWA aggregation for BUI inputs under simple uncertainty transformation will be omitted in this work.

\subsection{The multi-layer uncertainty transformation for BUI with corresponding OWA aggregation}

The proposal in this subsection needs the following definitions about the system of closed interval chains.

Definition 10. Given any closed interval $[a, b] \in \mathcal{I}$, its length is simply defined by a function $l: \mathcal{I} \rightarrow$ $[0,1]$ such that $l([a, b])=b-a$, i.e., the Lebesgue measure of $[a, b]$.
Remark. Observe that $l(S P(<x ; c>))=$ $l([c x, x+(1-c)(1-x)])=1-c$.

Definition 11. For any real number $x \in[0,1]$ and for any natural number $p \in\{1,2, \ldots\}$, a closed interval chain with degree (or layer) $p$ around $x$ is defined by a finite sequence $\left\{S P\left(<x ; h_{k}>\right)\right\}_{k=1}^{p}=$ $\left\{\left[h_{k} x, x+\left(1-h_{k}\right)(1-x)\right]\right\}_{k=1}^{p} \quad$ with $0 \leq h_{k} \leq$ $h_{k+1} \leq 1$ for any $k \in\{1, \ldots, p-1\}$. The space of all such closed interval chains with degree $p$ around $x$ is denoted by $\mathcal{I}^{<p ; x>}$.

Definition 12. A system of series with degree $p(p \in$ $\{1,2, \ldots\}), \mathbb{S}^{<p>}=\left\{\left\{h_{k}^{(\alpha)}\right\}_{k=1}^{p}\right\}_{\alpha \in[0,1]}$, is called an uncertain system, if it satisfies the following conditions:

(i) for any $0 \leq \alpha \leq 1$, it satisfies $\frac{1}{p} \sum_{k=1}^{p} h_{k}^{(\alpha)}=$ $\alpha$

(ii) for any $0 \leq \alpha<\beta \leq 1$ and any $k \in\{1, \ldots, p\}$, it holds $h_{k}^{(\alpha)} \leq h_{k}^{(\beta)}$.

Example 1. Consider $p=2$. If $\alpha \leq 0.5$, define $h_{1}^{(\alpha)}=0$ and $h_{2}^{(\alpha)}=2 \alpha$; if $\alpha>0.5$, define $h_{1}^{(\alpha)}=$ $2 \alpha-1$ and $h_{2}^{(\alpha)}=1$. Then, it can be verified that $\mathbb{S}^{<2>}=\left\{\left\{h_{1}^{(\alpha)}, h_{2}^{(\alpha)}\right\}\right\}_{\alpha \in[0,1]}=\{\{0,2 \alpha\}\}_{\alpha \in[0,0.5]} \cup$ $\{\{2 \alpha-1,1\}\}_{\alpha \in(0.5,1]}$ is an uncertain system with degree 2 .

With foregoing preparations, we next introduce a novel uncertainty transformation called the multilayer uncertainty transformation for BUI and the corresponding OWA aggregation.

Definition 13. The multi-layer uncertainty transformation for any BUI $<x ; c>$ with uncertain system $\mathbb{S}^{<p>}=\left\{\left\{h_{k}^{(\alpha)}\right\}_{k=1}^{p}\right\}_{\alpha \in[0,1]}$ is a mapping $M P: \mathcal{B} \rightarrow$ $\mathcal{I}^{<p ; x>}$ such that

$$
\begin{gathered}
M P(<x ; c>)=\left\{S P\left(<x ; h_{k}^{(c)}>\right)\right\}_{k=1}^{p} \\
=\left\{\left[h_{k}^{(c)} x, x+\left(1-h_{k}^{(c)}\right)(1-x)\right]\right\}_{k=1}^{p}
\end{gathered}
$$

Remark. Observe that $\frac{1}{p} \cdot \sum_{k=1}^{p} l\left(\operatorname{SP}\left(<x ; h_{k}^{(c)}>\right)\right)$ $=1-c$.

Example 2. Given BUI $<x ; c>=<0.4 ; 0.6>$ with uncertain system $\mathbb{S}^{<2>}=\left\{\left\{h_{1}^{(\alpha)}, h_{2}^{(\alpha)}\right\}\right\}_{\alpha \in[0,1]}=$ $\{\{0,2 \alpha\}\}_{\alpha \in[0,0.5]} \cup\{\{2 \alpha-1,1\}\}_{\alpha \in(0.5,1]}$, then

$$
\begin{aligned}
& M P(<0.4 ; 0.6>)=\left\{S P\left(<0.4 ; h_{k}^{(0.6)}>\right)\right\}_{k=1}^{2} \\
& =\{[(0.2)(0.4),(0.4)+(1-(0.2))(1-(0.4))],
\end{aligned}
$$

331 


$$
\begin{aligned}
& {[(1)(0.4),(0.4)+(1-1)(1-(0.4))]\} } \\
= & \{[0.08,0.88],[0.4,0.4]\} .
\end{aligned}
$$

Remark. Note that (11) implies that the average length of all involved intervals is equal to the uncertainty degree in that BUI $\langle x ; c\rangle$, i.e., $\frac{1}{p} \sum_{k=1}^{p} l\left(\left[h_{k}^{(c)} x, x+\left(1-h_{k}^{(c)}\right)(1-x)\right]\right)=1-c$. In addition, when $p=1$, the multi-layer uncertainty transformation in definition 13 degenerates into the simple uncertainty transformation in Definition 9.

Next, we define and illustrate the detailed steps of OWA aggregation for BUI with the multi-layer uncertainty transformation.

Definition 14. The OWA aggregation for BUI with the Multi-layer Uncertainty Transformation under RIM Quantifier $Q$ and uncertain system $\mathbb{S}^{<p>}=$ $\left\{\left\{h_{k}^{(\alpha)}\right\}_{k=1}^{p}\right\}_{\alpha \in[0,1]}$ (hereafter MUT_IOWA) is a function $M U T_{-} I O W A_{Q ; \mathbb{S}<p>}: \mathcal{B}^{n} \rightarrow \mathcal{I}$ whose function value is determined and obtained by the following course of actions from information preparation to final aggregation result returning, which in detail is separated into the following two stages containing 6 sub-steps in total.

Stage 1 Collect the overall presented inputs information for preference involved aggregation procedures.

Step 1: Fix $n(n \in\{2,3, \ldots\})$ and obtain BUI inputs $\left(<x_{i} ; c_{i}>\right)_{i=1}^{n}$.

Step 2: Select a RIM quantifier $Q$ as given preference for later generating weight vector.

Step 3: Fix a degree $p(p \in\{1,2, \ldots\})$ and determine an uncertain system with degree $p, \mathbb{S}^{<p>}=$ $\left\{\left\{h_{k}^{(\alpha)}\right\}_{k=1}^{p}\right\}_{\alpha \in[0,1]}$.

Step 4: For each $i \in\{1, \ldots, n\}$, using (11) to PERFORM the multi-layer uncertainty transformation for any BUI $<x_{i} ; c_{i}>$ with $\mathbb{S}^{<p>}$ and obtain

$$
\begin{aligned}
& M P\left(<x_{i} ; c_{i}>\right)=\left\{S P\left(<x_{i} ; h_{k}^{\left(c_{i}\right)}>\right)\right\}_{k=1}^{p} \\
& \quad=\left\{\left[h_{k}^{\left(c_{i}\right)} x_{i}, x_{i}+\left(1-h_{k}^{\left(c_{i}\right)}\right)\left(1-x_{i}\right)\right]\right\}_{k=1}^{p} .
\end{aligned}
$$

Stage 2 Perform corresponding IOWA aggregations and then take their average.

Step 5: For each $k \in\{1, \ldots, p\}$, perform IOWA operator with poset valued input vector

$X_{k}=\left(\left[h_{k}^{\left(c_{i}\right)} x_{i}, x_{i}+\left(1-h_{k}^{\left(c_{i}\right)}\right)\left(1-x_{i}\right)\right]\right)_{i=1}^{n} \in \mathcal{I}^{n}$

and inducing vector
$H_{k}=\left(\left[h_{k}^{\left(c_{i}\right)} x_{i}, x_{i}+\left(1-h_{k}^{\left(c_{i}\right)}\right)\left(1-x_{i}\right)\right]\right)_{i=1}^{n} \in \mathcal{I}^{n}$,

and obtain $I O W A_{Q ; H_{k}}\left(X_{k}\right)$ by Definition 7.

Step 6: Take an average and obtain a final aggregation result $M U T_{-} I O W A_{Q ; \mathbb{S}<p>}\left(\left(<x_{i} ; c_{i}>\right)_{i=1}^{n}\right)$ as follows, which embodies both the preferences involved and the uncertainty handled.

$$
\begin{aligned}
& M U T_{\_} I O W A_{Q ; \mathbb{S}<p>}\left(\left(<x_{i} ; c_{i}>\right)_{i=1}^{n}\right) \\
& =\frac{1}{p} \sum_{k=1}^{p} I O W A_{Q ; H_{k}}\left(X_{k}\right)
\end{aligned}
$$

Somewhat contradicting to normal intuition that MUT_IOWA might be monotonic with respect to $\preceq$, nevertheless, this is not the case. We neglect some further possible mathematical discussions since this study majorly focuses on the alternative methods to handle uncertainty in decision making.

\section{A numerical example of MUT_IOWA with application in financial evaluation}

Suppose a technical corporation needs to evaluate the success ratio of researching a new product. A management will firstly set a ratio threshold (e.g., 0.3 ) for further evaluation. Then, he will invite several consultants to give their opinions about the success ratio respectively, allowing uncertainties to be involved. Finally, if the aggregation result from consultants attains the threshold, the research plan can be approved.

In the next we illustrate the detailed aggregation process using MUT_IOWA, together with a simple decision making.

\section{The aggregation with uncertain information by using MUT_IOWA}

Stage 1 Collect the overall presented inputs information for preference involved aggregation procedures.

Step 1: Invite $n=3$ experts and require them to return the success ratios of the new research by BUI $<x_{1} ; c_{1}>=<0.5 ; 0.8>,<x_{2} ; c_{2}>=<$ $0.7 ; 1>$, and $<x_{3} ; c_{3}>=<0.8 ; 0.2>$, respectively.

Step 2: Select a RIM quantifier $Q$ with $Q(t)=t^{2}$, representing a pessimistic attitude of the management is involved. 
Step 3: Determine an uncertain system with degree $\quad p=2, \quad \mathbb{S}^{<2>}=\left\{\left\{h_{1}^{(\alpha)}, h_{2}^{(\alpha)}\right\}\right\}_{\alpha \in[0,1]}=$ $\{\{0,2 \alpha\}\}_{\alpha \in[0,0.5]} \cup\{\{2 \alpha-1,1\}\}_{\alpha \in(0.5,1]}$.

Step 4: For each $i \in\{1,2,3\}$, using (11) to perform the multi-layer uncertainty transformation for any BUI $<x_{i} ; c_{i}>$ with $\mathbb{S}^{<2>}$ and obtain

$$
\begin{aligned}
M P & \left(<x_{1} ; c_{1}>\right)=\left\{S P\left(<x_{1} ; h_{k}^{\left(c_{1}\right)}>\right)\right\}_{k=1}^{2} \\
= & \left\{\left[h_{k}^{\left(c_{1}\right)} x_{1}, x_{1}+\left(1-h_{k}^{\left(c_{1}\right)}\right)\left(1-x_{1}\right)\right]\right\}_{k=1}^{2} \\
= & \{[(0.6)(0.5),(0.5)+(1-0.6)(1-0.5)], \\
& {[(1)(0.5),(0.5)+(1-1)(1-0.5)]\} } \\
= & \{[0.3,0.7],[0.5,0.5]\} ; \\
M P( & \left.<x_{2} ; c_{2}>\right)=\left\{S P\left(<x_{2} ; h_{k}^{\left(c_{2}\right)}>\right)\right\}_{k=1}^{2} \\
= & \left\{\left[h_{k}^{\left(c_{2}\right)} x_{2}, x_{2}+\left(1-h_{k}^{\left(c_{2}\right)}\right)\left(1-x_{2}\right)\right]\right\}_{k=1}^{2} \\
= & \{[(1)(0.7),(0.7)+(1-1)(1-0.7)], \\
& {[(1)(0.7),(0.7)+(1-1)(1-0.7)] } \\
= & \{[0.7,0.7],[0.7,0.7]\} ; \\
M P & \left(<x_{3} ; c_{3}>\right)=\left\{S P\left(<x_{3} ; h_{k}^{\left(c_{3}\right)}>\right)\right\}_{k=1}^{p} \\
= & \left\{\left[h_{k}^{\left(c_{3}\right)} x_{3}, x_{3}+\left(1-h_{k}^{\left(c_{3}\right)}\right)\left(1-x_{3}\right)\right]\right\}_{k=1}^{2} \\
= & \{[(0)(0.8),(0.8)+(1-0)(1-0.8)], \\
& {[(0.4)(0.8),(0.8)+(1-0.4)(1-0.8)]\} } \\
= & \{[0,1],[0.32,0.92]\} .
\end{aligned}
$$

Stage 2 Perform corresponding IOWA aggregations and then take their average.

Step 5: For each $k \in\{1,2\}$, by (12) and (13), perform IOWA operator with poset valued input vector $X_{k}=\left(\left[h_{k}^{\left(c_{i}\right)} x_{i}, x_{i}+\left(1-h_{k}^{\left(c_{i}\right)}\right)\left(1-x_{i}\right)\right]\right)_{i=1}^{n} \in$ $\mathcal{I}^{3}$ and inducing vector

$H_{k}=\left(\left[h_{k}^{\left(c_{i}\right)} x_{i}, x_{i}+\left(1-h_{k}^{\left(c_{i}\right)}\right)\left(1-x_{i}\right)\right]\right)_{i=1}^{3} \in \mathcal{I}^{3}$ In detail, $X_{1}=([0.3,0.7],[0.7,0.7],[0,1])$ and $X_{2}=([0.5,0.5],[0.7,0.7],[0.32,0.92]), \quad$ and inducing vector $H_{k}$ is same to $X_{k}$, $H_{1}=X_{1}=([0.3,0.7],[0.7,0.7],[0,1]) \quad$ and $H_{2}=X_{1}=([0.5,0.5],[0.7,0.7],[0.32,0.92])$.

Next, by Definition 7 (omitting the detailed computation for weight vector $w$ ), we have

$$
I O W A_{Q ; H_{1}}\left(X_{1}\right)=(2 / 9)([0.3,0.7])+(4 / 9)([0.7,0.7])
$$$$
+(1 / 3)([0,1])=[0.378,0.8],
$$

$$
\begin{array}{r}
I O W A_{Q ; H_{2}}\left(X_{2}\right)=(2 / 9)([0.5,0.5])+(4 / 9)([0.7 \\
0.7])+(1 / 3)([0.32,0.92])=[0.5289,0.7289] .
\end{array}
$$

Step 6: Take an average and obtain a final aggregation result by (14),

$$
\begin{aligned}
& \text { MUT_IOWA } \\
& =\frac{1}{2} \sum_{k=1}^{2} I_{\mathbb{S}<2>}\left(\left(<x_{i} ; c_{i}>H_{k}\left(X_{k}\right)=(0.5)([0.378,0.8])\right.\right. \\
& +(0.5)([0.5289,0.7289])=[0.45345,0.76445]
\end{aligned}
$$

Stage 3 A direct decision taking using information obtained from previous aggregations.

Step 7: Since the predetermined threshold is 0.3 and $[0.45345,0.76445]>[0.3,0.3]$, then the decision is made to approve the research plan.

It is noteworthy that in real decision making, above Stage 3 can be extended and improved by decision makers according to their different decision backgrounds and rules.

\section{Conclusions}

Real value based aggregation functions are fundamental and powerful tools in evaluation and decision making theories and practices, but they cannot directly deal with the inputs (e.g., BUI) that involve uncertainties. When the given inputs are BUI, this study discussed the methods to handle them by proposing some uncertainty transformations for them, returning some intervals numbers which can be easier to be further tackled and aggregated.

To fulfill this aim, based on some previous studies and results, we firstly introduced IOWA operator with poset valued input vector and inducing vector. Subsequently, we defined the concept of uncertain system, based on which we further proposed the multi-layer uncertainty transformation for BUI. Then, with such transformation, we formally introduced MUT_IOWA aggregation procedure, which contains two stages along with six detailed steps.

Rather than with only one fixed uncertainty transformation which tends to be simplistic and lack flexibility in real decision making, the uncertain system introduced in Definition 12 provides more 
diversity in modeling uncertainties of decision makers and therefore helps to build the multi-layer uncertainty transformation which is the basis of MUT_IOWA aggregation procedure.

Some numerical examples helped to understand the usage of MUT_IOWA, and introduced its further applications in decision making. The proposed models in this study also provide some more diversity in aggregation theory. In further studies, we will also concentrate on a more complex decision making scenario where some second-order preference will be considered.

\section{Acknowledgments}

This work is partly supported under Scientific Research Start-up Foundation with Grant 184080H202B165; partly supported from the Science and Technology Assistance Agency under contract No. APVV-17-0066; partly supported from the project of Grant Agency of the Czech Republic (GAČR) no. 18-06915S; partly supported from the Natural Science Foundation of Jiangsu Province (Grants No BK20150870 and No. BK20190695); partly supported from the Jangsu's Philosophy and Social Science Fund (grant no. 19GLC010).

\section{References}

[1] G. Choquet. Theory of capacities, Ann. Inst. Fourier 5 (1954) 131-295.

[2] H. Bustince, et al., A historical account of types of fuzzy sets and their relationships, IEEE Trans. Fuzzy Syst. 24(1) (2016) 179-194.

[3] M. Grabisch, J.L. Marichal, R. Mesiar and E. Pap, Aggregation Functions, Cambridge University Press (2009), ISBN:1107013429.

[4] L. Jin, Eliciting and measuring hesitance in decisionmaking, Int. J. Intell. Syst. 34(6) (2019) 1206-1222.

[5] L. Jin, M. Kalina, R. Mesiar and S. Borkotokey, Certainty aggregation and the certainty fuzzy measures, Int. J. Intell. Syst. 33(4) (2018) 759-770.

[6] L. Jin, M. Kalina, R. Mesiar and S. Borkotokey, Discrete choquet integrals for Riemann integrable inputs with some applications, IEEE Trans. Fuzzy Syst. 26(5) (2018) 31643169.

[7] L. Jin, R. Mesiar and G. Qian, Weighting models to generate weights and capacities in multi-criteria group decision making, IEEE Trans. Fuzzy Syst. 26(4) (2018), 2225-2236.
[8] L. Jin, R. Mesiar and R.R. Yager, Ordered weighted averaging aggregation on convex poset, IEEE Trans. Fuzzy Syst. 27(3) (2019), 612-617.

[9] L. Jin, R. Mesiar and R.R. Yager, Melting probability measure with OWA operator to generate fuzzy measure: the crescent method, IEEE Trans. Fuzzy Syst. 27(6) (2019), 1309-1316.

[10] E.P. Klement and R. Mesiar, Discrete integrals and axiomatically defined functionals, Axioms 1(1) (2012), 9-20.

[11] E.P. Klement, R. Mesiar and E. Pap, Triangular norms, Springer-Verlag, Berlin, Heidelberg, 2000.

[12] X.W. Liu and Q.L. Da, A decision tree solution considering the decision maker's attitude, Fuzzy Sets Syst. 152 (2005), 437-454.

[13] X.W. Liu and S.L. Han, Orness and parameterized RIM quantifier aggregation with OWA operators: A summary, Int. J. Approx. Reasoning 48 (2008) 77-97.

[14] B. Llamazares, Choosing OWA operator weights in the field of social choice, Inf. Sci. 177 (2007), 4745-4756.

[15] J.M. Merigo and A.M. Gil-Lafuente, New decision-making techniques and their application in the selection of financial products, Inf. Sci. 180 (11) (2010), 2085-2094.

[16] R. Mesiar, S. Borkotokey, L. Jin and M. Kalina, Aggregation functions and capacities, Fuzzy Sets Syst. 346(1) (2018), 138-146.

[17] R. Mesiar, L. Sipeky, P. Gupta and L. Jin, Aggregation of OWA operators, IEEE Trans. Fuzzy Syst. 26(1) (2018), 284 291.

[18] R. Mesiar, S. Borkotokey, L. Jin and M. Kalina, Aggregation under uncertainty, IEEE Trans. Fuzzy Syst. 26(4) (2018), 2475-2478.

[19] X. Pu, R. Mesiar, R.R. Yager and L. Jin, Fuzzy integral logic and interval sugeno integral with preference, IEEE Trans. Fuzzy Syst. DOI: 10.1109/TFUZZ.2019.2908127

[20] M. Sugeno, Theory of fuzzy integrals and its applications, $\mathrm{PhD}$ thesis. Tokyo Institute of Technology (1974).

[21] V. Torra and Y. Narukawa, The interpretation of fuzzy integrals and their application to fuzzy systems, Int. J. Approx. Reasoning 41 (2006) 43-58.

[22] R.R. Yager, On ordered weighted averaging aggregation operators in multicriteria decision making, IEEE Trans. Syst. Man Cybern. 18(1) (1988), 183-190.

[23] R.R. Yager, Quantifier guided aggregation using OWA operators, Int. J. Intell. Syst. 11 (1996), 49-73.

[24] R.R. Yager and D.P. Filev, Induced ordered weighted averaging operators, IEEE Trans. Syst. Man Cybern. 29 (1999), 141-150.

[25] R.R. Yager, J. Kacprzyk and G. Beliakov, Recent developments on the ordered weighted averaging operators: Theory and practice, Springer-Verlag, Berlin, 2011.

[26] F.J. Cabrerizo, M.R. Ureña, W. Pedrycz and E. HerreraViedma, Building consensus in group decision making with an allocation of information granularity, Fuzzy Sets Syst. 255 (2014), 115-127. 